\title{
Die Echte Rentierflechte (Cladonia rangiferina) und ihre Verwandtschaft
}

\author{
Götz Heinrich Loos
}

\begin{abstract}
Reindeer lichen (Cladonia rangiferina) is a light-coloured, fruticose lichen belonging to the Cladoniaceae. Cladonia rangiferina often dominates the ground in boreal pine forests and open, low-alpine sites in a wide range of habitats, from humid, open forests, rocks and heaths. In Alaska the Dena ina also boil reindeer lichen and drink the juice as a medicine for diarrhea. Biology, ecology, distribution and uses of reindeer lichen are outlined.
\end{abstract}

\section{Zusammenfassung}

Rentierflechte (Cladonia rangiferina) ist eine hellgraue Flechte aus der Familie der Cladoniaceae. Die Rentierflechte ist oft eine dominierende Art auf dem Boden borealer Nadelwälder. Sie kommt in verschiedenen Lebensräumen vor, an feuchten Standorten, in lichten Wäldern, auf Felsen und in Heidevegetation. In Alaska kochen Ureinwohner (Dena ina) die Rentierflechte auf und trinken den dabei entstehenden Saft als Mittel gegen Durchfall. Biologie, Ökologie, Verbreitung und Verwendung der Rentierflechte werden erläutert.

\section{Strauchflechten (Cladonia)}

Die Strauchflechten-Gattung Cladonia umfasst nach ihrem Gestaltbau mehrere Typen, namentlich die Becher-, Säulen- und Rentierflechten. Der Thallus (oder deutsch: das Lager) ist der Körper der Flechten. Er ist bei den Cladonien, wie die Cladonia-Arten allgemein eingedeutscht genannt werden, auffallend gestaltet: Am bekanntesten dürfte die „Becherform“ sein, bei welcher ein Teil des Thallus zu einem auffälligen, aufrechten becherartigen Podetium entwickelt ist, an dem sich die Fruchtkörper (Apothecien) ausbilden können. Ziemlich häufig aus dieser Gruppe ist vor allem die Gewöhnliche Becherflechte (Cladonia fimbriata), die auf fast jedem alten Weidezaunpfosten und Holzstapel wächst. Bei den Säulenflechten ist das Podetium schmal, stab-, säulen- oder spindelförmig, unverzweigt oder mit eher etwas unregelmäßigen Verzweigungen versehen. Die deutschlandweit häufigste derartige Cladonie ist die Gewöhnliche Säulenflechte (Cladonia coniocraea), vielfach an ähnlichen Standorten wie die erwähnte Becherflechte, aber mehr noch an mindestens etwas feuchten, oft bemoosten Stammfüßen von Bäumen; im Ruhrgebiet ist sie die häufigste Cladonie insgesamt, was auch für andere Regionen zutreffen mag. Die dritte Gruppe schließlich bilden Rentierflechten, die aufrechte, reich verzweigte Stämmchen aufweisen, die an ein unbelaubtes Bäumchen oder ein übermäßig gegliedertes Geweih erinnern. Sie werden auch als eigene Gattung Cladina in einigen Bearbeitungen abgetrennt, sind aber doch mit Cladonia so eng verwandt, dass sie in jüngster Zeit kaum noch als eigene Gattung akzeptiert werden (vgl. Munger 2008 und dort genannte Literatur), sondern Cladina als Untergattung von Cladonia geführt wird (siehe z. B. ESSLINGER 2014).

\section{Rentierflechte als Futter und Nahrung}

Der Name Rentierflechte bezieht sich jedoch weniger auf die Gestalt als auf die Tatsache, dass diese in Skandinavien besonders in der nördlichen (borealen) Nadelwaldzone und in der Tundra weit verbreitet ist und einen Hauptbestandteil der Nahrung für Rentiere insbesondere im Winter ausmacht (so benötigen Rentiere angeblich $2 \mathrm{~kg}$ Trockengewicht Flechten täglich und können in Gefangenschaft nur überleben, wenn ihnen Flechten in die Nahrung beigegeben werden, wie man u.a. auf vielen Internetseiten nachlesen kann; entsprechende Auskünfte erteilten aber auch mehrere Zoos). Elche und Moschusochsen in der hohen Arktis ernähren sich ebenfalls im Winter fast ausschließlich von Rentierflechten. Für die menschliche Ernährung hat die Rentierflechte keine wirkliche Bedeutung, dennoch wurde sie in Notzeiten gesammelt und gegessen. Der bittere Geschmack ist allerdings sehr gewöh- 


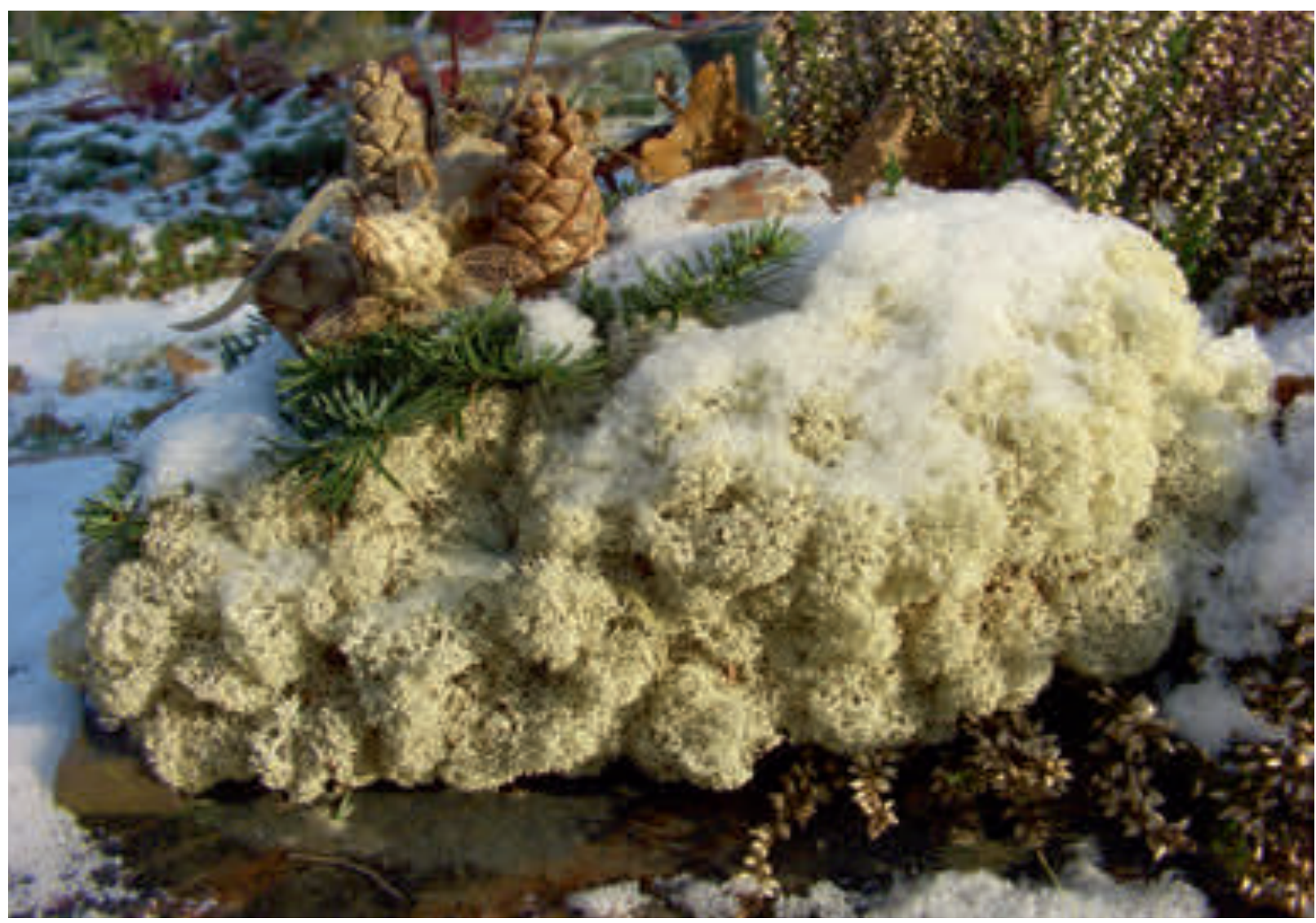

nungsbedürftig. Volkstümlich wird ihr bisweilen der Name „Isländisch Moos“ zugeschrieben, der sich jedoch korrekterweise auf die Flechtenart Cetraria islandica bezieht.

\section{Verwendung von Rentierflechten}

Da Letztere als Arzneipflanze genutzt wurde, gab es in den Zeiten der Volksmedizin früher auch Verwechslungen, und statt dem echten Isländisch Moos wurden Rentierflechten eingesetzt; über die Wirkungen ist allerdings nichts bekannt. Der Name Isländisch Moos oder Rentierflechte wird auch bei Modellbauern, z. B. für Modellbahnlandschaften, benutzt, bezieht sich jedoch in aller Regel auf eine weitere Art, die Alpen-Rentierflechte (Cladonia stellaris), die sehr viel zartere Stämmchen aufweist, die außerdem halbkugelig oder kuppelförmig vorgewölbte Endabschnitte aufbauen. Dies ist auch die verbreitete Flechte (oft als „Moos“ tituliert) in Grabgestecken und Weihnachtsdekoration;

Abb. 3: Cladonien werden häufig in der Trauerfloristik verwendet. sie wird zu diesem Zweck in großen Mengen aus Skandinavien exportiert. Auch der Echten Rentierflechte und weiteren Arten kommt gelegentlich eine ähnliche Verwendung zu oder sie sind der Alpen-Rentierflechte beigemischt.

\section{4. Äußeres Erscheinungsbild und Bestimmungsmerkmale}

Die Echte Rentierflechte hebt sich vom Untergrund durch ihre grauweiße, helle, matte Farbe, die nur an den Astspitzen nach braun bis braunviolett umschlägt, deutlich ab. Darin ist sie relativ konstant und unterscheidet sich dadurch von den meisten anderen Rentierflechtenarten, die überwiegend etwas grauer, brauner oder grünlicher sind. Die Äste der Stämmchen sind an der Spitze einseitswendig, von einer Verzweigungsstelle gehen drei oder vier Äste ab. Blättchen und Schuppen fehlen ganz (auch bodenständige Blättchen sind nicht vorhanden), lediglich die Stämmchenoberfläche wirkt bei näherer Betrachtung etwas filzig. Eine Absicherung der Bestimmung erfolgt auf chemischem Wege: Bei Beträufelung mit Kalilauge färbt sich die Ober- 

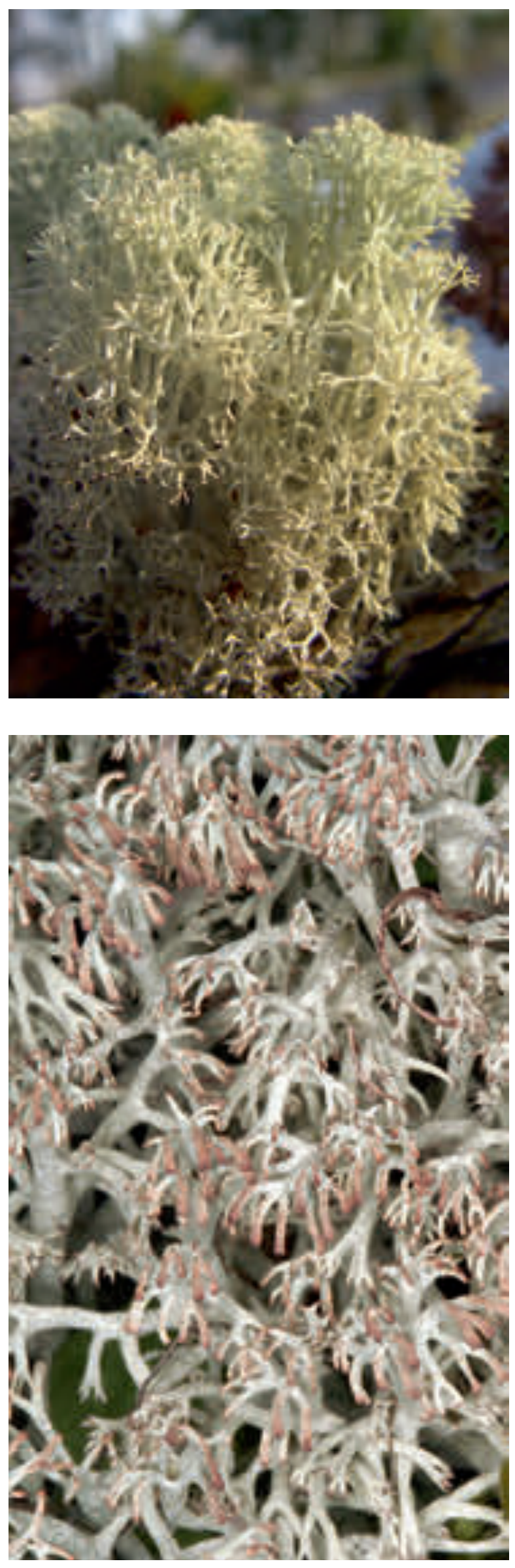

fläche der Stämmchen und Äste gelb oder zumindest schwach gelblich; nimmt man paraPhenylendiamin-Lösung, ergibt sich eine hellrote bis orange Färbung. Fruchtkörper (Apothecien) bildet die Echte Rentierflechte nur selten aus.

\section{Standort, Verbreitung und Gefährdung}

Die Echte Rentierflechte ist in der Tundrenzone eine typische und überall häufige, massenhafte Erscheinung der flechtenreichen Rasen und geht weit nach Norden in arktische Gebiete, sowohl in Eurasien als auch in Nordamerika. Darüber hinaus existieren Vorkommen im Himalaja und in tropischen Gebirgen. In Deutschland ist die Art selten und als stark gefährdet eingestuft. Sie ist gesetzlich besonders geschützt und findet sich im Anhang $\mathrm{V}$ der FFH-Richtlinie. Ihre Verbreitung in der Bundesrepublik ist ausführlicher bei WIRTH $\&$ al. (2013) behandelt, demnach meidet sie Trockengebiete fast gänzlich, kommt dagegen in den kühl-feuchten Lagen praktisch sämtlicher Mittelgebirge und der Alpen vor - oder besser gesagt, in einigen dieser Gebiete kam sie vor, hat vielfach hohe Bestandseinbußen hinnehmen müssen bis hin zum völligen Verschwinden. Die Art soll in manchen Regionen jedoch früher sehr häufig gewesen sein (vgl. z. B. Loos 2010 zum Vorkommen in Westfalen). Ihre Bestandssituation in Hessen haben Cezanne \& al. (2009a, b) ausführlich behandelt; hier zeigt sie Verbreitungsschwerpunkte in Kellerwald, Ostsauerländer Gebirgsrand, Taunus, Sandsteinodenwald, Rhön und Hohem Meißner, tiefländische Vorkommen sind hingegen nicht mehr existent, was auch sonst für Deutschland weitestgehend gilt. Die Echte Rentierflechte besiedelt lückige Zwergstrauchheiden, darunter die Hochheiden der Mittelgebirge - insbesondere des Rothaargebirges an der hessisch-westfälischen Grenze - Sandmagerrasen, offene

\section{Abb. 2 (oben): Cladonia stellaris.}

Abb. 3 (unten): Ausschnitt aus dem Flechtenkörper von Cladonia rangiferina.

Abb. 4 (Seite 157): Skandinavischer Schmuck, der Becherflechten nachempfunden wurde. 
Borstgrasrasen, nicht zu nasse Stellen in Hochmooren, lichte Silikatfelsstandorte wie Blockhalden sowie bodensaure, magere Waldstandorte, vor allem in kühl-feuchten Lagen. Damit kommt sie überwiegend an vom Menschen geschaffenen Standorten vor und leidet heute darunter, dass es die traditionellen Nutzungsformen, auch der Wälder, kaum noch gibt. Derartige Vorkommen scheinen daher nur in Naturschutzgebieten gesichert, in denen entsprechende Nutzungen noch imitiert werden, um diese und andere Arten sowie Biotopstrukturen $\mathrm{zu}$ erhalten. Besonders bedroht ist die Echte Rentierflechte durch die allgemeine Nährstoffanreicherung (Hypertrophierung) der Landschaft und verschwindet bei Verdichtung der Vegetation relativ schnell. Viele ehemalige Standorte sind heute vergrast, verbuscht oder durch Stickstoff bevorzugende Pflanzen eingenommen, in Ackerland umgewandelt oder durch Anlage von Forsten bzw. andere Formen der ertragserzielenden Waldwirtschaft zu sehr beschattet. Sollten die Prognosen zum Klimawandel zutreffen, muss mit einer Austrocknung von Wuchsorten gerechnet werden, die diese Art aller Voraussicht nach nicht überleben wird.

\section{Verwandte Arten}

Mit der Echten Rentierflechte eng verwandt sind weitere Arten. Außer der genannten Alpen-Rentierflechte kommen in Deutschland aus der Untergattung Cladina (Rentierflechten im eigentlichen Sinne) und nahe stehenden Gruppen noch mindestens 11 zusätzliche Arten vor. Unter ihnen ist die variable, wohl noch aufzugliedernde Gruppe der Gabel-Cladonie (Cladonia furcata), die von allen noch am weitesten verbreitet ist und auch regelmäßiger an trockeneren Standorten, so auf Brachen in städtischindustriellen Räumen und Bahngelände, vorkommt. Ihre Verzweigung ist weniger „harmonisch“ regelmäßig als bei den eigentlichen Rentierflechten. Aus Hessen sind nach Cezanne \& al. (2009b) aus der eigentlichen Rentierflechtengruppe weitere 4 Arten sowie der Cladonia-squarrosa-mitis-Artenkomplex gemeldet, von denen eine (die oben erwähnte Alpen-Rentierflechte) verschollen ist.

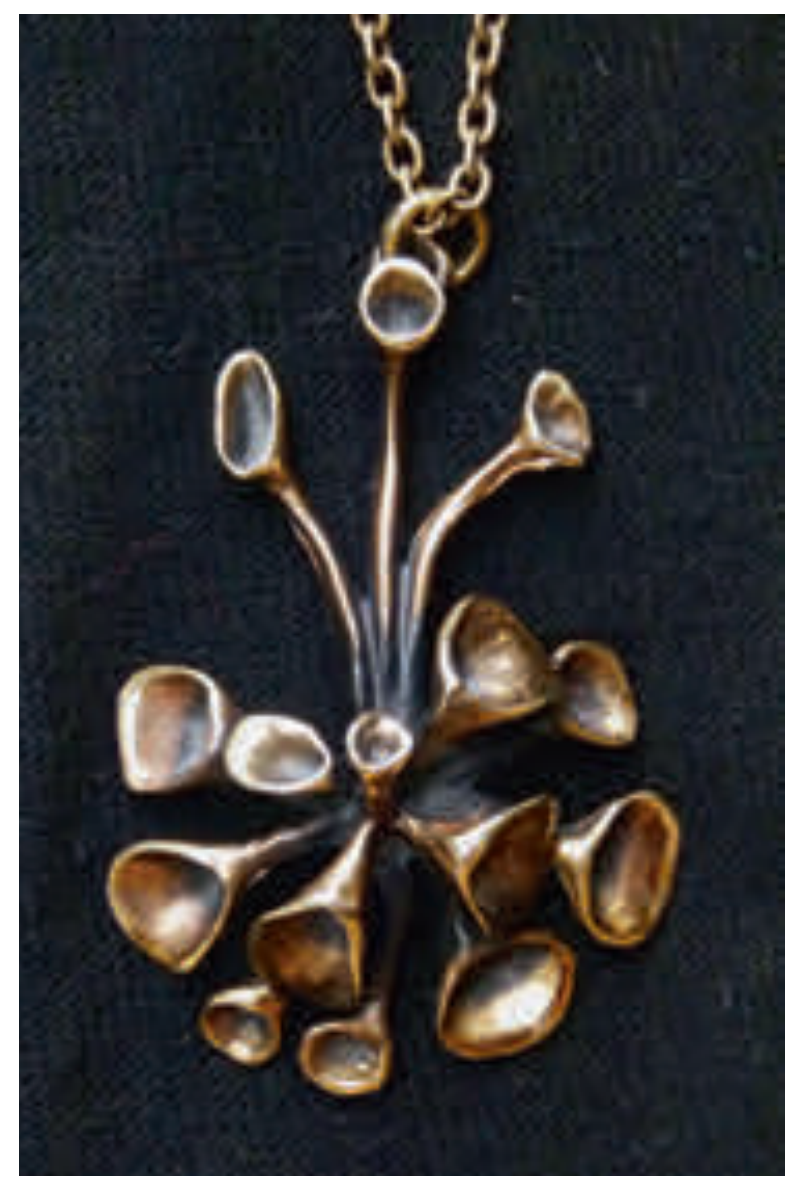

\section{Literatur}

Cezanne, R., Eichler, M., Hohmann, M.-L. \& Teuber, D. 2009a: Artensteckbrief Cladonia rangiferina (L.) Weber ex F. H. Wigg., Art der FFH-Richtlinie Anhang V. - Hessen-Forst, FENA-Schriftenreihe.

Cezanne, R., Eichler, M., Hohmann, M.-L. \& Teuber, D. 2009b: Gutachten zur gesamthessischen Situation der Rentierflechten, Gattung Cladonia L. subgenus Cladina (NYL.) VAIN, Arten des Anhangs V der FFH-Richtlinie. 2. Fassung. Hessen-Forst, FENA-Schriftenreihe. - Gießen. Loos, G. H. 2010: Pflanzenporträt: Cladonia rangiferina - Echte Rentierflechte (Cladoniaceae), Flechte des Jahres. - Jahrbuch des Bochumer Botanischen Vereins 1: 207-208. Wirth, V., Hauck, M. \& Schultz, M. 2013: Die Flechten Deutschlands. Band 1. - Stuttgart.

\section{Internetseiten}

EsSLINGER, T. 2014: A cumulative checklist for the lichen-forming, lichenicolous and allied fungi of the continental United States and Canada. Version 19. - North Dakota State University.

http://www.ndsu.edu/pubweb/ - esslinge/chcklst/chcklst7. htm

Munger, G. T. 2008: Cladonia spp. In: Fire effects information system. - U.S. Department of Agriculture, Forest Service, Rocky Mountain Research Station, Fire Sciences Laboratory (Producer). http://www.fs.fed.us/ database/feis/ [30.11.2014] 